\title{
An Epidemiological Observation of Judicious use of Antibiotics in Dhar District, M.P. (India)
}

\author{
Rekha Bisht*, Arvind Dabi, Pankaj Dixit, Dinesh Kumar Mishra \\ Department of Pharmacology, Indore Institute of Pharmacy, Opposite IIM, Rau-Pithampur Road, Indore. Madhya Pradesh, INDIA.
}

\begin{abstract}
Background: Antibiotic resistance is presently the gigantic challenge to the effective treatment of infections globally. It is a rapidly growing clinical problem and public health threat. Promiscuous use of antibiotics is epidemic in India and this is an affair of consequential concern. Irrational use of antibiotics can cause increase adverse drug reaction, lead to antibiotic resistance and increase the treatment cost. Antibiotic resistance unfavourably influence both clinical and financial therapeutic results, with repercussion ranging from the failure of an individual patient to respond to therapy and the need for costly and/or toxic alternative medicine to the social cost of higher morbidity and mortality rates, longer duration of hospitalization and the need for changes in empirical therapy. Analysing the antibiotics utilization pattern is significant in the context of its escalating use and its overall impact on the health care system. Materials and Methods: An observational and prospective study was conducted by interviewing (using a data collection form) the patients to evaluate the utilization of antibiotics in multispecialty hospital, Dhar district, Madhya Pradesh, India. Results: Out of 102 patients interviewed, 85 (83.33\%) were prescribed antibiotics either alone or in combination with other drugs. The maximum number of patients received antibiotics belongs to the age group of $31-40(22,18.7 \%)$. Ciprofloxacin $(25,21.25 \%)$ was the widely prescribed antibiotics followed by metronidazole $(20,17 \%)$ penicillin $(15,12.75 \%)$, azithromycin (12, 10.2\%) cephalosporin $(11,9.35 \%)$ and amoxicillin $(10,8.5 \%)$. Maximum prescriptions of antibiotics were for high grade fever $(29,24.65 \%)$ followed by gastrointestinal infection $(18,15.3 \%)$. Conclusion: Proper strategy like antibiotic policy and educational intercession are necessary to control the excessive use of antibiotics in health care settings.
\end{abstract}

Key words: Antibiotics, Drug utilization, Antibiotic Resistance, Observational study, Prospective study.

\section{INTRODUCTION}

Antibiotics are among the most widely prescribed medications both in the hospital setting and the community setting. Resistance to antibiotics becomes a major threat to public health due to escalating consumption of antibiotic. ${ }^{1}$ Bacterial resistance to antibiotics is an increasing clinical issue worldwide and estimated to cause 10 million deaths annually by $2050 .^{2,3}$ Excessive use of antibiotic is a primary driver of antibiotic resistance and reducing antibiotic use is a central strategy for confronting resistance. ${ }^{4}$ There are various reasons for escalating use of antibiotics includes rising incomes, health insurance and burden of infectious worldwide. ${ }^{5}$ In the United States, over 2 million people are affected with antibiotic-resistant infections each year, account for at least 23,000 deaths and have a total economic burden that exceeds $\$ 20$ billion in direct healthcare costs alone. ${ }^{6}$ In India, $20 \%$ to $50 \%$ of all antibiotics used are appearing to be used not only in excess but also inappropriately. ${ }^{7}$ If the current scenario does not change, there will be economic losses of 100 trillion dollars due to resistant infections worldwide. ${ }^{8}$

Because of high treatment costs associated with the resistant infections and limited access to antibiotics, India is assailable to the loss of antibiotic efficacy. ${ }^{9}$ It has been estimated that by 2050, 700,000 deaths per year occur inevitably to antimicrobial resistance and, there might be 10 million deaths per year. ${ }^{8}$ Bacteria causing common or severe infections have developed resistance to varying degrees to each new antibiotic coming to market since over several decades.
DOI: 10.5530/ijopp.14.2.19

Address for correspondence: Dr. Rekha Bisht, Associate Professor, Department of Pharmacology, Indore institute of Pharmacy, Madhya Pradesh, INDIA. Phone no: +919752095118 Email id: rekha_al03@rediffmail. com

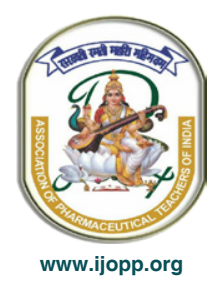


Faced with this reality, the call for action to prevent a developing global crisis in health care is essential. ${ }^{10} \mathrm{~A}$ prerequisite for appropriate use of antibiotics and limited spread of antibiotic resistance is the proper knowledge about the judicious use of these life-saving drugs. ${ }^{11}$

A rational use of antibiotics is vital to diminish the emergence of antibiotic resistance and to extend the useful lifetime of effective antibiotics. ${ }^{12}$ Escalating the knowledge and practice of judicious use of antibiotics among primary care physicians is one way to reduce antibiotic resistance. ${ }^{13}$ In order to identify the proper utilization of antibiotics, an audit of prescriptions of inpatients and outpatients was undertaken at Govt. District Hospital, multispecialty hospital in Dewas, Madhya Pradesh, India.

\section{MATERIALS AND METHODS}

\section{Study Type}

A cross sectional and prospective study was conducted to evaluate the utilization of antibiotics in Dhar district, Madhya Pradesh, India. The study was conducted by interviewing the in-patients and outpatients and studying their prescriptions. The patient fulfilling the inclusion criteria were selected and evaluated for epidemiological and the prescribing patterns of antibiotics. The permission to carry out the study was approved by the Hospital's Chief Medical Officer; Letter No. 3597-98 dated 06/08/19.

\section{Study Site}

The study was carried out in Govt. District Hospital (Mahatma Gandhi Hospital) Dewas, a multispecialty hospital, one of the major health care hospitals providing both IPD and OPD services for many disease treatment in Dewas, Madhya Pradesh.

\section{Study Setting}

The study was performed using prescriptions of 102 patients of both IPD and OPD. Patients of various age groups admitted in different wards of hospital were selected for the study to collect the data. The prospective study in various patients was conducted who had received one or more course of treatment with antibiotics. Demographic details of patient's such as the age, name, gender, socioeconomic status, social habits and data on prescribed drugs that include name of drug, dosage form, route of administration, most widely prescribed drugs and patient's characteristics, laboratory investigations and clinical data were obtained from the hospital records. Peculiar data on different categories like service, concomitant disease and drug therapy, type of infection and antibiotics prescribed were collected by using a structured data collection form in an approved manner. Patients of various age groups admitted in hospital excluding children below the age group of 10 , pregnant women and older patient above the age group of 80 , were selected for the study.

\section{RESULTS}

Out of 102 patients interviewed, 85 were prescribed antibiotics either as monotherapy or in combination with other drugs. Gender wise distribution of patients revealed that, 57 patients were male and 43 were females accounting for $58.14 \%$ and $43.86 \%$ of total population interviewed for the study. The maximum number of patients who were prescribed antibiotics was between the age groups of $31-40(22,18.7 \%)$ and $51-60(20,17 \%)$ followed by 41-50 (15, 12.75\%), 21-30 (11, 9.35\%), 61-70 (08, 6.8\%), 71-80 (05, 4.25\%) and 10-20 (04, 3.4\%). Amongst the various reasons for administration of antibiotics, the maximum reasons were of high grade fever $(29,24.65 \%)$ and GIT infection $(18,15.3 \%)$ followed by respiratory tract infection $(12,10.2 \%)$, injury cases $(8,6.8 \%)$, urinary tract infection $(8,6.8 \%)$, ascites $(5,4.25 \%)$ (Table 1). The maximum number of antibiotic prescribed were ciprofloxacin $(25,21.25 \%)$ and metronidazole $(20,17 \%)$ followed by penicillin $(15,12.75 \%)$, cephalosporin $(11,9.35 \%)$, azithromycin $(12,10.2 \%)$ and amoxicillin (10, 8.5\%) (Table 2).

\section{DISCUSSION}

The present study revealed that large number of antibiotics was prescribed to the patients in Govt. District Hospital, Dewas. Maximum number of antibiotics was prescribed in the patients between the age group of 31-40. Maximum antibiotics were prescribed in patients with high grade fever and GIT infection.

\begin{tabular}{ccc}
\hline \multicolumn{2}{c}{ Table 1: Reason for administration of antibiotics. } \\
\hline Reason & $\begin{array}{c}\text { Number of } \\
\text { Patients }\end{array}$ & Percentage (\%) \\
\hline High grade fever & 29 & 24.65 \\
GIT infection & 18 & 15.3 \\
Respiratory tract & 12 & 10.2 \\
infection & & \\
$\quad$ Injury & 8 & 6.8 \\
Urinary Tract & 8 & 6.8 \\
Infection & & \\
Ascites & 5 & 4.25 \\
Others & 5 & 4.25 \\
Total & $\mathbf{8 5}$ & $\mathbf{1 0 0 \%}$ \\
\hline
\end{tabular}




\begin{tabular}{|c|c|c|}
\hline $\begin{array}{l}\text { Antibiotics } \\
\text { Prescribed }\end{array}$ & $\begin{array}{l}\text { Number of } \\
\text { Patients }\end{array}$ & Percentage (\%) \\
\hline Ciprofloxacin & 25 & 21.25 \\
\hline Metronidazole & 20 & 17 \\
\hline Penicillins & 15 & $12.75 \%$ \\
\hline Cephalosporins & 11 & $9.35 \%$ \\
\hline Azithromycin & 12 & 10.2 \\
\hline Amoxicillin & 10 & 8.5 \\
\hline Total & 85 & $100 \%$ \\
\hline
\end{tabular}

Ciprofloxacin was the most widely prescribed antibiotic followed by metronidazole either in combination or alone. The escalating frequency with which antibiotic resistant microorganisms are recovered from patients in hospital and community setting has been discussed widely in recent years. ${ }^{7}$ To slow the development of antibiotic resistance in bacteria and to extend the useful lifetime of effective antibiotics, it is essential to promote judicious use of antibiotics. ${ }^{12}$ The major driving force in increasing the inappropriate use of antibiotics is the excessive prescription by general practitioners, even in the absence of appropriate indications. In addition, diagnostic uncertainty and self-medication often encourages over-prescription especially when the clinical symptoms of viral or bacterial aetiology are similar. ${ }^{10}$ The major selective pressure driving changes in the frequency of likelihood of resistance is in each case, the volume of antibiotics use. ${ }^{7}$ Continuous and proper monitoring of antimicrobial use and knowledge of prescription pattern are some of the strategies recommended to subdue resistance to antimicrobials in hospitalized patients. ${ }^{14}$

\section{CONCLUSION}

Antibiotic resistance is rapidly escalating global health problem. It contributes to health and economic losses worldwide to a great extent. Escalating rates of antibiotic resistance has been suggested by research studies in countries with high per-capita antibiotic consumption. In order to restraint antibiotic resistance, socio-economic factors like access to clean water and sanitation, regulation of private healthcare sector and better governance are equally important along with diminishing the antibiotic consumption. As antibiotics have crucial role in clinical care, thus efforts should be made to decline the volume of unnecessary antibiotic prescribing and necessary counselling should be done to reduce the self-medication. Upgrading the quality, not just the quantity of medication will need professional and public education towards judicious use of antibiotics. Advancement and assessment of medical and veterinary practice guidelines, preferable diagnostic tests and curtailment of antibiotic use as growth booster in food and animals, development of new antibiotics are some of the important steps required. The consequences of antibiotic resistance are affecting everybody in the world. To prove the forecast of an approaching post-antibiotic era wrong, the time has come to strengthen research to identify cost-effective strategies and drastically ameliorate our antibiotic prescribing practices for controlling resistance. Reducing the inappropriate use of antibiotics in outpatient settings is utmost important requirement which will require the development of strategies that address both patient and provider level factors that impact prescribing practices and ultimately supports in reducing the likelihood of resistance to these lifesaving drugs.

\section{CONFLICT OF INTEREST}

The authors declare no conflict of interest.

\section{ABBREVIATIONS}

IPD: In Patient Department; OPD: Out Patient Department; GIT: Gastrointestinal infection.

\section{SUMMARY}

An observational and prospective study was conducted by interviewing (using a data collection form) the patients to evaluate the utilization of antibiotics in multispecialty hospital, Dhar district, Madhya Pradesh, India. Total 102 patients were interviewed. The results showed that $85(83.33 \%)$ patients were prescribed antibiotics either alone or in combination with other drugs. The maximum number of patients received antibiotics belongs to the age group of $31-40(22,18.7 \%)$. Ciprofloxacin was the widely prescribed antibiotics followed by metronidazole, penicillin, azithromycin, cephalosporin and amoxicillin. Maximum prescriptions of antibiotics were for high grade fever followed by gastrointestinal infection. To control the likelihood of antibiotic resistance, educational intervention and antibiotic policy are utmost important requirement in the present scenario.

\section{REFERENCES}

1. Randad RD, Bhagwate ST, Inamdar MK. Drug utilization study of some antibiotic in indoor setting at tertiary care teaching hospital in central India: A descriptive study. Int J Basic Clin Pharmacol. 2017;5(6):1123-6.

2. Schechner V, Temkin E, Harbarth S, Carmeli Y, Schwaberb MJ. Epidemiological interpretation of studies examining the effect of antibiotic usage on resistance. Clin Microbiol Rev. 2013;2(26):289-307. 
3. Dixit A, Kumar N, Kumar S, Trigun V. Antimicrobial resistance: Progress in the decade since emergence of New Delhi metallo- $\beta$-lactamase in India Indian J Community Med. 2019;1(44):4-8.

4. Olesen SW, Barnett ML, MacFadden DR, Brownstein JS, Díaz SH, Lipsitch $\mathrm{M}$, et al. The distribution of antibiotic use and its association with antibiotic resistance. ELife. 2018;7:e39435.

5. Singh SK, Sengupta AS, Bhattacharya M, Ramasubramanian A, Sharma $\mathrm{S}$, Sahu S, et al. Variations in antibiotic use across India: Multi-centre study through global point prevalence survey. J Hosp Infect. 2019;3(103):280-3.

6. Davis ME, Liu TL, Taylor YJ, Davidson L, Schmid M, Yates T, et al. Exploring patient awareness and perceptions of the appropriate use of antibiotics: A mixed-methods study. Antibiotics. 2017;6(4):23.

7. Bisht R, Katiyar A, Singh R, Mittal P. Antibiotic resistance: A global issue of concern. Asian J Pharm Clin Res. 2009;2(2):34-9.

8. Pereira JQ, Silva MT, Galvao TF. Use of antibiotics by adults: A populationbased cross-sectional study. Sao Paulo Med J. 2018;136(5):407-13.
9. Farooqui HH, Selvaraj S, Mehta A, Heymann DL. Community level antibiotic utilization in India and its comparison vis-à-vis European countries: Evidence from pharmaceutical sales data. PLoS One. 2018;13(10):e0204805.

10. Prestinaci F, Pezzotti P, Pantosti A. Antimicrobial resistance: A global multifaceted phenomenon. Patho Glob Health. 2015;109(7):309-18.

11. Marit W, Abdifatah A, Røen IL, Karoline E, Tijana S, Kjell H, et al. Knowledge of antibiotics and antibiotic resistance among Norwegian pharmacy customers: A cross-sectional study. BMC Public Health. 2019;19(1):66.

12. Atti MLC, Amore CD, Ceradini J, Paolini V, Ciliento G, Chessa G, et al. Prevalence of antibiotic use in a tertiary care hospital in Italy, 2008-2016. Ital J Pediatr. 2019;45(1):63.

13. Chang Y, Chusri S, Sangthong R, McNeil E, Hu J, Du W. Clinical pattern of antibiotic overuse and misuse in primary healthcare hospitals in the southwest of China. PLoS One. 2019;14(6):e0214779.

14. Norberto K, El-Ahal WA, Lavie YB, Salim H. Antibiotic prescription and cost patterns in a general intensive care unit. Pharm Pract. 2007;5(2):67-73. 